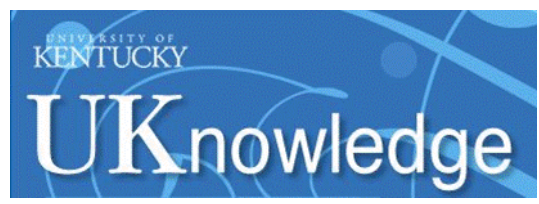

University of Kentucky

UKnowledge

Information Science Faculty Publications

Information Science

5-2-2019

\title{
Sensitive Research, Practice and Design in $\mathrm{HCl}$
}

Stevie Chancellor

Georgia Tech

Nazanin Andalibi

University of Michigan

Lindsay Blackwell

University of Michigan

David Nemer

University of Kentucky, david.nemer@uky.edu

Wendy Moncur

University of Dundee, UK

Follow this and additional works at: https://uknowledge.uky.edu/slis_facpub

Part of the Graphics and Human Computer Interfaces Commons, and the Library and Information

Science Commons

Right click to open a feedback form in a new tab to let us know how this document benefits you.

\section{Repository Citation}

Chancellor, Stevie; Andalibi, Nazanin; Blackwell, Lindsay; Nemer, David; and Moncur, Wendy, "Sensitive Research, Practice and Design in HCl" (2019). Information Science Faculty Publications. 85.

https://uknowledge.uky.edu/slis_facpub/85

This Conference Proceeding is brought to you for free and open access by the Information Science at UKnowledge. It has been accepted for inclusion in Information Science Faculty Publications by an authorized administrator of UKnowledge. For more information, please contact UKnowledge@lsv.uky.edu. 


\section{Sensitive Research, Practice and Design in $\mathrm{HCl}$}

\section{Digital Object Identifier (DOI)}

https://doi.org/10.1145/3290607.3299003

\section{Notes/Citation Information}

Published in CHIEA '19, paper no. W33.

(C) 2019 Copyright held by the owner/author(s)

Permission to make digital or hard copies of part or all of this work for personal or classroom use is granted without fee provided that copies are not made or distributed for profit or commercial advantage and that copies bear this notice and the full citation on the first page. Copyrights for third-party components of this work must be honored. For all other uses, contact the owner/author(s). 


\title{
Sensitive Research, Practice, and Design in $\mathrm{HCl}$
}

\author{
Stevie Chancellor \\ Georgia Tech \\ Atlanta, GA, USA \\ schancellor3@gatech.edu \\ Lindsay Blackwell \\ University of Michigan \\ Ann Arbor, MI, USA \\ lblackw@umich.edu \\ Wendy Moncur \\ University of Dundee \\ Dundee, UK \\ wmoncur@dundee.ac.uk
}

\author{
Nazanin Andalibi \\ University of Michigan \\ Ann Arbor, MI, USA \\ andalibi@umich.edu \\ David Nemer \\ University of Kentucky \\ Lexington, KY, USA \\ David.nemer@uky.edu
}

\section{ABSTRACT}

New research areas in $\mathrm{HCl}$ examine complex and sensitive research areas, such as crisis, life transitions, and mental health. Further, research in complex topics such as harassment and graphic content can leave researchers vulnerable to emotional and physical harm. There is a need to bring researchers together to discuss challenges across sensitive research spaces and environments. We propose a workshop to explore the methodological, ethical, and emotional challenges of sensitive research in $\mathrm{HCl}$. We will actively recruit from diverse research environments (industry, academia, government,

Permission to make digital or hard copies of part or all of this work for personal or classroom use is granted without fee provided that copies are not made or distributed for profit or commercial advantage and that copies bear this notice and the full citation on the first page. Copyrights for third-party components of this work must be honored. For all other uses, contact the owner/author(s).

CHI'19 Extended Abstracts, May 4-9, 2019, Glasgow, Scotland Uk

() 2019 Copyright held by the owner/author(s).

ACM ISBN 978-1-4503-5971-9/19/05.

https://doi.org/10.1145/3290607.3299003 
etc.) and methods areas (qualitative, quantitative, design practices, etc.) and identify commonalities in and encourage relationship-building between these areas. This one-day workshop will be led by academic and industry researchers with diverse methods, topical, and employment experiences.

\section{CCS CONCEPTS}

- Human-centered computing $\rightarrow \mathrm{HCl}$ theory, concepts and models;

\section{KEYWORDS}

Sensitive research; ethics; vulnerable populations

\section{ACM Reference Format:}

Stevie Chancellor, Nazanin Andalibi, Lindsay Blackwell, David Nemer, and Wendy Moncur. 2019. Sensitive Research, Practice, and Design in $\mathrm{HCl}$. In CHI Conference on Human Factors in Computing Systems Extended Abstracts (CHI'19 Extended Abstracts), May 4-9, 2019, Glasgow, Scotland Uk. ACM, New York, NY, USA, 8 pages. https://doi.org/10.1145/3290607.3299003

\section{INTRODUCTION}

In $\mathrm{HCl}$, researchers and practitioners are increasingly exploring sensitive contexts [3, 13, 24]. The growth of research in crisis [22], designing for life transitions [12, 20], difficult life events or stigmatized experiences [2, 8, 17], and mental health challenges [6, 19, 23] highlight growing interest around complex human experiences. Further, new research areas investigate graphic content [6, 19] with methods from machine learning [5, 6] to action research [7] and content analysis [2, 19], exposing researchers to large volumes of disturbing content. As AI and data-driven efforts push to solve pernicious problems like scrubbing graphic content from websites, industry professionals and data scientists grapple with these topics to design effective platform tools.

These research contexts also highlight noticeable risks to researchers themselves [16], an area which Andalibi and Forte have described as "researchers as vulnerable populations" [1]. Qualitative research and fieldwork have the potential to negatively impact the researcher's emotional and physical health and safety [4], as is the case in crisis work in the field. Even if the research is not conducted in person, scholars across disciplines risk facing online abuse, whether due to their research topics or their personal identities [3, 15, 16, 18].

Frequently, these areas overlap, and research in a context that is emotionally sensitive for participants is also sensitive for the researcher - putting both participants and researchers at risk. Traditional forms of governance for research do not answer questions about appropriate methods, ethics, and protection for such sensitive research. Protections of researchers themselves are often left out of review protocols [16], if the protocol is not already exempt from ethics board approval (as is the case for much research on public or corporate data). Researchers collaborate across methods or topical 
areas, and their knowledge and experiences flow across these partnerships and discussions through formal collaboration and informal networking groups. As interest in workshops related to this have shown $[3,13,24], \mathrm{HCl}$ more broadly recognizes the importance of working through sensitive contexts.

However, there is a need and value for those who engage in sensitive research to interact with each other in formal settings, both to build stronger connections and share best practices as well as to bring in those who do not have existing support structures at their home institutions or companies. In contrast to previous workshops, we intend to connect researchers across topics, methods domains, and research institutions (academic, industry, government, etc.) to discuss commonalities between sensitive research domains in $\mathrm{HCl}$. These discussions are critical for the sharing of best practices and the formation and establishment of recommendations in our community and beyond. Additionally, sensitive research topics demand unique collaborations across methods and topics areas to resolve complexity and, in some cases, to reduce risk.

We propose a one-day workshop to explore sensitive research in $\mathrm{HCl}$, both for sensitive topics as well as sensitivity to researcher well-being and safety. Our workshop will support 25-30 participants across topical and methodological areas of research, design, and practice. In addition to topic areas mentioned before, we are also interested in recruiting industry perspectives and quantitative researchers who may lack support and guidance in their day-to-day work. Broadly, our goals are to build support networks for researchers in these sensitive research areas; to share knowledge and experience from past and current work; to facilitate connections between academic researchers and industry professionals; and to share concrete insights and open questions with the $\mathrm{CHI}$ community.

\section{CORE GOALS}

\section{During}

- Build support networks across researchers of sensitive domains to share experiences. We will accomplish this through interactive exercises like Researcher Speed Dating and small group activities.

- Exchanging knowledge and experiences from past and ongoing research to inform current and future research goals. We will accomplish this through group work, a poster during $\mathrm{CHI}$, and the dissemination of a public cheat sheet or written publication summarizing out findings.

- Enable new collaborations across three areas of interests: methodological collaborations, topical foci, and intersections between industry and academic research. We will accomplish this through our recruitment strategies.

\section{After/Post-Workshop Plans}


- Share with $\mathrm{CHI}$ conference participants. Participants will collaboratively draft and design a Poster that, with approval from the Posters and Workshop Chairs, we will present at one of the poster plenaries. The organizers have done this with success at past workshops [19]. We also see this as an important outreach technique to connect with researchers in sensitive contexts who may not be able to attend or participate in the workshop due to travel, funding, or employment constraints.

- Share with the broader $\mathrm{HCl}$ community. We will let workshop participants choose between four potential public-facing venues to share our ideas publicly: a CACM article; a journal submission an Interactions magazine piece; a series of blog posts on Medium; or a summary of the topics discussed as a publicly available "playbook" available for download on our website. Participants will choose whether or not to associate their names with any public document, should some participants wish to participate anonymously.

- Facilitate post-workshop support. We will create a private messaging group through a platform such as Slack or Discord for participants to share new research, reach out for support, and continue conversations after the workshop ends.

\section{ORGANIZERS}

This group of workshop organizers was selected to reflect the diversity we hope to recruit for participants as well as diversity in methods and topical expertise. Organizers have successfully (co-)organized workshops in the past at CHI [3, 14], CSCW [21], NordiCHI [13], GROUP [11], and ICWSM [9, 10].

Stevie Chancellor is a PhD Candidate in Human Centered Computing at Georgia Tech. She researches data-driven methods to understand deviant mental health behavior in online communities, and combines techniques from Natural Language Processing, Machine Learning, and Data Science to understand sensitive health behaviors. Her research focuses on identifying and predicting content from pro-eating disorder communities on social media.

Nazanin Andalibi, Ph.D is a Research Fellow/Research Investigator at the University of Michigan's School of Information. She uses mixed methods and theorizes social media behaviors such as sensitive disclosures and interactions around them, and provides designs and recommendations for social technologies that foster human well-being. She concentrates on forms of human suffering that can be isolating and lead to distress, such as abuse, mental illness, and pregnancy loss.

Lindsay Blackwell is a PhD candidate in the School of Information at the University of Michigan and a UX Researcher with PRO Unlimited at Facebook. She uses mixed methods to investigate online harassment, including the experiences and support needs of harassment targets and the motivations of people who participate in abusive behaviors online. Her dissertation research focuses on motivating bystanders to intervene in online harassment without risking their own comfort and safety. 


\section{WORKSHOP RULES}

Chatham House rules Due to the sensitive and risky nature of the research area, we will use Chatham House Rules: participants can discuss and use any information learned in the workshop, but they cannot reveal who shared it or the names and affiliations of any individual participants. This is particularly important for industry participants, who need protection to discuss material they encounter in their professional experiences.

No Judgment Breaks + Downtime Built into Schedule The topics discussed in this workshop may be emotionally exhausting and in some cases graphic. We will encourage participants to step out of the room, walk around, or take a break if they feel overwhelmed. Further, we are not scheduling work through coffee breaks or lunch to encourage participants to take legitimate breaks from what can be difficult subjects.
David Nemer, Ph.D is an Assistant Professor in the School of Information Science at the University of Kentucky. His research and teaching interests cover the intersection of ICT for Development (ICT4D), science and technology studies (STS), and human-computer interaction (HCI). Nemer is an ethnographer who is specifically interested in studying ICTs in less industrialized parts of the world to understand the effects of ICTs on the development and empowerment of marginalized communities.

Wendy Moncur, Ph.D is Interdisciplinary Professor of Digital Living at the University of Dundee. She is also a Visiting Scholar at the University of Technology Sydney, Australia, and an Associate of the Centre for Death and Society. Her research focuses on being human in a Digital Age. She has examined the design and use of technology in becoming a parent, relationship breakdown, retiral and end of life. She also examines methodological issues around the conduct of research in sensitive contexts, and opportunities for research to illuminate policy.

\section{LOGISTICS}

Website. A website, www.sensitivehci.com, will be used to solicit submissions for the workshop and as a repository for materials. This will include the Call for Papers, resources, position papers, schedule adjustments, and post-workshop reports. If participants choose not to publicly share their submissions, we will share it only with other workshop participants via email.

Recruitment. We will promote the workshop through a variety of social media channels and mailing lists (Facebook CHI Meta page; Researchers of the Socio-Technical Facebook Page; recruitment on Twitter); mailing lists (such as the AoIR List and the local SICSA-HCI [Scottish $\mathrm{HCI}$ ] mailing list); by contacting participants of past workshops related to this subject [3,9-11,13]; and by contacting industry practitioners directly (Google, Facebook, Twitter, Reddit, etc.).

Submissions. Position papers should be 2-4 pages and should take a position on sensitive research in $\mathrm{HCl}$. This includes current research and works in progress in a sensitive domain, reflections on the research process or lessons learned, or emergent challenges in these research domains. Position papers are certainly not limited to these subjects, and we encourage novel conceptualizations of the space of sensitive research.

Position papers should also include a brief description of participants' experiences with sensitive research, as either a participant, researcher, or both, and what they hope to gain from participating in this workshop.

Participants will be selected based on their prior experience and interest in the workshop as well as the quality of their submissions. We will focus on recruiting from a diverse group of participants (balance of graduate students and faculty; industry practitioners and academic audiences; representation of different cultures, genders, and races).

Materials. This workshop will require chairs and tables for 30 total participants + organizers, with 6-8 participants per table; a podium for the keynote and a microphone for accessibility; a projector 


\section{SCHEDULE}

9am-9:20: Welcome and introduction 9:20-10:05: Keynote and Q\&A

10:10-11: Researcher speed dating

11am: Coffee break

11:15-12:15pm: Pre-organized group work

12:15: All hands

1-2:30: Lunch

2:30-3:30: Emergent areas group work

3:30pm: Coffee break

3:45-4:25: All hands

4:30-5pm: Moving forward

$5 \mathrm{pm}$ : End workshop

6pm: Post-workshop dinner and a projection screen (optional but nice if the room has it); paper pad and markers for collaborative brainstorming; and cardstock for participants to make name tents.

\section{BREAKDOWN OF ACTIVITIES}

Welcome and Introduction Organizers will introduce the workshop and the themes of the day, and share the ground rules of the workshop for all participants. We will also share a shared Google Doc to collect ideas from participants.

Researcher Speed Networking Participants will line up and get 60 seconds to introduce themselves to another member of the workshop as well as a brief description of their research and what they hope to get out of participation. This will serve as an ice breaker activity for the participants to begin to get to know each other.

Keynote Pending acceptance of the workshop, we will recruit a keynote speaker for a 20-25 minute talk on their expertise with sensitive research. In the unlikely event that we're not able to secure a keynote speaker for the workshop, we will expand the Speed Dating and Pre-Organized Group Work sections.

Pre-Organized Group Work We will break the group into working groups, organized by submissions. In this group work session, we'll work on networking group members, focusing on diversity of the written statements.

All Hands Both All-Hands sessions will bring the group together and collaboratively brainstorm on the emergent issues from group work. These will be used to spur further group discussion about these issues.

Emergent Areas Group Work Workshop participants reorganize into smaller groups by interest in high-level challenge groups brainstormed before lunch (4-5 groups). Small group work on these challenges.

Moving Forward Going forward, we answer the question in the group: how do we share this information with others? Iterate and finalize poster draft and decide on best publication medium for the findings of our research.

\section{CALL FOR PAPERS}

We invite submissions to "Sensitive Research, Practice, and Design in $\mathrm{HCl}$," a one day workshop at $\mathrm{CH}$ in Glasgow, UK on May 5, 2019. Our workshop explores the methodological, ethical, and emotional challenges of sensitive research in $\mathrm{HCl}$, both for participants and for researchers and practitioners who conduct this work.

We invite current research and works in progress in a sensitive domain, reflections on the research process and emergent challenges. Topics of interest include but are not limited to: 
- Current research on a sensitive domain

- Issues of recruitment and consent

- Methods selections and analysis

- Privacy, anonymity, and risk management

- Best practices to support those conducting sensitive research

- Reflections on past research

Position papers should also include a brief description of their experiences with sensitive research, as either a participant, researcher, or both, and what they hope to gain from participating in this workshop.

All submissions should be 2-4 pages (excluding references), and submitted in CHI EA format (old or new). Submit your position papers to sensitivehci2019@gmail.com by February 12, 2019. Papers will be peer-reviewed by the workshop committee, and chosen for their relevance to the workshop topic, clarity of their position paper, and the overall composition of the workshop. At least one author of each accepted position paper must attend the workshop, and all participants must register for both the workshop and for at least one day of the conference.

More details about the workshop can be found at www.sensitivehci.com. We look forward to your submission.

\section{REFERENCES}

[1] Nazanin Andalibi and Andrea Forte. 2015. Social Computing Researchers as Vulnerable Populations. In ACM Conference on Computer Supported Cooperative Work \& Social Computing Workshop on Ethics for Studying Sociotechnical Systems in a Big Data World.

[2] Nazanin Andalibi and Andrea Forte. 2018. Announcing Pregnancy Loss on Facebook: A Decision-Making Framework for Stigmatized Disclosures on Identified Social Network Sites. In Proceedings of the 2018 CHI Conference on Human Factors in Computing Systems. ACM, 158

[3] Lindsay Blackwell, Mark Handel, Sarah T Roberts, Amy Bruckman, and Kimberly Voll. 2018. Understanding Bad Actors Online. In Extended Abstracts of the 2018 CHI Conference on Human Factors in Computing Systems. ACM, W21.

[4] Daniela K Busse, Alan Borning, Samuel Mann, Tad Hirsch, Lisa P Nathan, Andrea Grimes Parker, Ben Shneiderman, and Bryan Nunez. 2013. CHI at the barricades: an activist agenda?. In CHI'13 Extended Abstracts on Human Factors in Computing Systems. ACM, 2407-2412.

[5] Stevie Chancellor, Yannis Kalantidis, Jessica A Pater, Munmun De Choudhury, and David A Shamma. 2017. Multimoda Classification of Moderated Online Pro-Eating Disorder Content. In Proceedings of the $2017 \mathrm{CHI}$ Conference on Human Factors in Computing Systems. ACM, 3213-3226.

[6] Stevie Chancellor, Zhiyuan Lin, Erica L Goodman, Stephanie Zerwas, and Munmun De Choudhury. 2016. Quantifying and predicting mental illness severity in online pro-eating disorder communities. In Proceedings of the 19th ACM Conference on Computer-Supported Cooperative Work \& Social Computing. ACM, 1171-1184.

[7] Rachel Clarke, Peter Wright, Madeline Balaam, and John McCarthy. 2013. Digital portraits: photo-sharing after domestic violence. In Proceedings of the SIGCHI conference on Human factors in computing systems. ACM, 2517-2526. 
[8] Jill P Dimond, Casey Fiesler, and Amy S Bruckman. 2011. Domestic violence and information communication technologies. Interacting with Computers 23, 5 (2011), 413-421.

[9] Casey Fiesler, Stevie Chancellor, Anna Lauren Hoffmann, Jessica Pater, and Nicholas John Proferes. 2016. Challenges and Futures for Ethical Social Media Research. In AAAI Conference on Web and Social Media (ICWSM): Workshop.

10] Casey Fiesler, Stevie Chancellor, Katie Shilton, Jessica Vitak, and Michael Zimmer. 2018. Exploring Ethical Trade-Offs in Social Media Research. In AAAI Conference on Web and Social Media (ICWSM): Workshop.

[11] Casey Fiesler, Pamela Wisniewski, Jessica Pater, and Nazanin Andalibi. 2016. Exploring Ethics and Obligations for Studying Digital Communities. In Proceedings of the 19th International Conference on Supporting Group Work. ACM, 457-460.

[12] Oliver L Haimson, Jed R Brubaker, Lynn Dombrowski, and Gillian R Hayes. 2015. Disclosure, stress, and support during gender transition on Facebook. In Proceedings of the 18th ACM Conference on Computer Supported Cooperative Work \& Social Computing. ACM, 1176-1190.

[13] Daniel Herron, Nazanin Andalibi, Oliver Haimson, Wendy Moncur, and Elise van den Hoven. 2016. $\mathrm{HCl}$ and sensitive life experiences. In Proceedings of the 9th Nordic Conference on Human-Computer Interaction. ACM, 134.

[14] Neha Kumar, Kurtis Heimerl, David Nemer, Naveena Karusala, Aditya Vashistha, Susan M Dray, Christian Sturm, Laura S Gaytán-Lugo, Anicia Peters, Nova Ahmed, et al. 2018. HCl Across Borders: Paving New Pathways. In Extended Abstracts of the 2018 CHI Conference on Human Factors in Computing Systems. ACM, Sym03.

[15] Alice E Marwick, Lindsay Blackwell, and Katherine Lo. 2016. Best practices for conducting risky research and protecting yourself from online harassment (Data \& Society Guide). New York: Data and Society Institute (2016).

[16] Wendy Moncur. 2013. The emotional wellbeing of researchers: considerations for practice. In Proceedings of the SIGCHI Conference on Human Factors in Computing Systems. ACM, 1883-1890.

[17] Fayika Farhat Nova, Md Rashidujjaman Rifat, Pratyasha Saha, Syed Ishtiaque Ahmed, and Shion Guha. 2018. Silenced Voices: Understanding Sexual Harassment on Anonymous Social Media Among Bangladeshi People. In Companion of the 2018 ACM Conference on Computer Supported Cooperative Work and Social Computing. ACM, 209-212.

[18] Cristhian Parra, David Nemer, David Hakken, and Vincenzo D’Andrea. 2015. Deep Trust in the future of Community Informatics. The Journal of Community Informatics 11, 2 (2015).

[19] Jessica Pater and Elizabeth Mynatt. 2017. Defining Digital Self-Harm. In Proceedings of the 2017 ACM Conference on Computer Supported Cooperative Work and Social Computing. ACM, 1501-1513.

[20] Corina Sas and Steve Whittaker. 2013. Design for forgetting: disposing of digital possessions after a breakup. In Proceedings of the SIGCHI Conference on Human Factors in Computing Systems. ACM, 1823-1832.

[21] Austin Toombs, Laura Devendorf, Patrick Shih, Elizabeth Kaziunas, David Nemer, Helena Mentis, and Laura Forlano. 2018 Sociotechnical Systems of Care. In Companion of the 2018 ACM Conference on Computer Supported Cooperative Work and Social Computing. ACM, 479-485.

[22] Sarah Vieweg, Amanda L Hughes, Kate Starbird, and Leysia Palen. 2010. Microblogging during two natural hazards events: what twitter may contribute to situational awareness. In Proceedings of the SIGCHI conference on human factors in computing systems. ACM, 1079-1088.

[23] Rui Wang, Min SH Aung, Saeed Abdullah, Rachel Brian, Andrew T Campbell, Tanzeem Choudhury, Marta Hauser, John Kane, Michael Merrill, Emily A Scherer, et al. 2016. CrossCheck: toward passive sensing and detection of mental health changes in people with schizophrenia. In Proceedings of the 2016 ACM International Joint Conference on Pervasive and Ubiquitous Computing. ACM, 886-897.

[24] Jenny Waycott, Hilary Davis, Anja Thieme, Stacy Branham, John Vines, and Cosmin Munteanu. 2015. Ethical encounters in $\mathrm{HCl}$ : Research in sensitive settings. In Proceedings of the 33rd Annual ACM Conference Extended Abstracts on Human Factors in Computing Systems. ACM, 2369-2372. 